\title{
Electron-LA Phonon Interaction in a Quantum Dot
}

\author{
T. EZAKI*, N. MORI and C. HAMAGUCHI \\ Department of Electronic Engineering, Osaka University, Japan 2-1 Yamada-oka, \\ Suita, Osaka 565, Japan
}

\begin{abstract}
Relaxation time due to electron-longitudinal-acoustic (LA) phonon interaction is calculated in a GaAs quantum dot (QD) with $N$ electrons (from $N=1$ to 4 ), where electrons in a narrow quantum well are confined by a parabolic confining potential, by using the exact eigen states of electrons. Although the energy levels become dense with increasing the number of electrons, the modification of the relaxation time is found to be not so strong, which attributes the fact that many electron eigen states consist of only a few dominant single electron states which limit the electron relaxation. By comparing relaxation process via intermediate states with the direct process, several fastest processes are found to be realized by relaxation through intermediate states between the initial state and the ground state. The effect of change in the quantum well width is also discussed.
\end{abstract}

Keywords: Electron-phonon interaction, quantum dot, phonon bottleneck, exact diagonalization method, Coulomb interaction

\section{INTRODUCTION}

Low dimensional semiconductor structures such as quantum dots (QDs) have attracted much attention because of their unique features in electronic properties [1-3] and their applications to future electron devices [4,5]. Although their quantized atomic like energy levels are expected to have advantages for optical devices, energy relaxation via electron-optical-phonon interaction is diminished in QDs for energy level separation of order 1 meV because the mismatch between the energy level separation and the optical-phonon energy of, for example, $36 \mathrm{meV}$ in the bulk GaAs [6-10], which is often referred as "phonon bottleneck". Several theoretical and experimental approaches have been devoted to the study of the phonon bottleneck and pointed out a possibility to avoid it by utilizing Auger processes [11], multiphonon processes [12] or the role of excited energy levels of the exciton [13].

In the process of light emission, electrons and holes are first created in higher energy states and relax down to the ground state in cascade via

\footnotetext{
* Correpsonding author.
} 
phonon emissions and finally recombine to emit light. Energy relaxations from higher energy levels to sublevels near the ground state are expected to be fast because optical-phonon interaction plays a dominant role in the electron relaxation. At the final stage of electron relaxation toward the ground state, electron-longitudinal-acoustic (LA) phonon interaction is expect to be important because of the small energy separation compared to the optical-phonon energy. In a QD containing a few electrons with dot size comparable to the effective Bohr radius, many electron eigen states are considerably modified by the Coulombic interaction between the electrons. In order to investigate electron-phonon interaction in such a QD, we therefore take into account the exact $N$ electron eigen states (for $N$ up to 4) by numerically diagonalizing the complete $N$ particle Hamiltonian [14-16] including the Coulombic interaction between electrons and then calculate electron-LA phonon relaxation rates toward the ground state using the $N$ particle eigen states. Since there are many intermediate states between the initial state and the ground state, we calculate relaxation time via intermediate states in order to find the fastest process.

\section{EIGEN STATES OF A MODEL QD}

We consider a QD fabricated on a $\mathrm{AlGaAs} / \mathrm{GaAs} /$ AlGaAs quantum well structure as a model system. The confining potential of a circular QD in $x-y$ plane parallel to the hetero-interface is modeled by an isotropic and parabolic potential $\frac{1}{2} m^{*} \omega_{0}\left(x^{2}+y^{2}\right)$ with $\hbar \omega_{0}$ being the single electron confining energy. Throughout this paper we assume a typical value of $\hbar \omega_{0}=1 \mathrm{meV}$ for the parabolic potential. The Hamiltonian of $N$ electrons including the Coulomb interaction between the electrons can be written as

$$
\mathcal{H}=\sum_{i=1}^{N} \mathcal{H}_{0 i}+\sum_{i<j} \frac{e^{2}}{4 \pi \varepsilon\left|r_{i}-r_{j}\right|}
$$

$$
\mathcal{H}_{0 i}=-\frac{\hbar^{2}}{2 m^{*}} \nabla_{i}^{2}+\frac{1}{2} m^{*} \omega_{0}^{2}\left(x_{i}^{2}+y_{i}^{2}\right)+V\left(z_{i}\right)
$$

where $m^{*}$ is the effective mass of an electron. Along the $z$ direction, we assume an infinite square well potential of the width $W$ and consider only the ground state associated with the quantized $z$ motion, because electrons are frozen out into the lowest subband in experimentally realized dots.

In order to solve the $N$ particle Hamiltonian, we use $N$ particle Slater determinants as the basis sets which can be composed by the solutions of the single particle Hamiltonian $\mathcal{H}_{0 i}$. The many particle eigen functions are then obtained by diagonalizing the many particle Hamiltonian. Figure 1 shows the lowest 40 eigen energies of a QD with single, two and three electrons. For a single electron QD, equally separated energy levels are seen, which reflects the single-electron confining energy $\hbar \omega_{0}=1 \mathrm{meV}$. When more electrons are added into the QD, total ground state energy of the system moves up and becomes greater than the simple sum of the single-electron energy. Moreover the energy level structure becomes more dense with increasing the number of electrons. From this analysis the Coulomb interaction is expected to play an very important role in determining the energy spectra in a few electron system in a QD $[1,16]$.
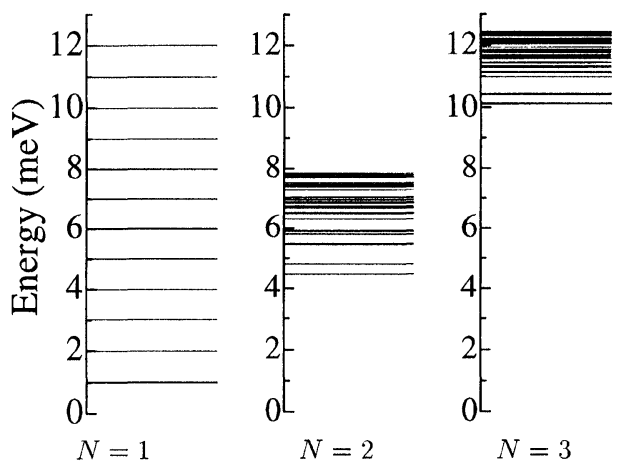

FIGURE 1 Total eigen energies in a QD which contains single, two and three electrons. In the model used in the present study, energy level separation for single electron QD is $\hbar \omega_{0}=1 \mathrm{meV}$. For a QD with two and three electrons, energy level spectra become to form more complex and dense structures due to the Coulombic interaction. 


\section{ELECTRON-LA PHONON INTERACTION}

Since we are interested in energy relaxation of electrons in QDs at low temperatures, we take into account only the phonon emission process and thus assume the lattice temperature $T=0 \mathrm{~K}$ for simplicity. Since the maximum energy separation in our model system is the order of $1 \mathrm{meV}$, electron-optical-phonon relaxation can be ignored and the electron-LA phonon interaction dominates the relaxation processes. The electron-LA phonon scattering rate $W_{i i^{\prime}}$ derived from the firstorder perturbation theory using the Fermi's golden rule is described by the following relation

$W_{i i^{\prime}}=\frac{2 \pi}{\hbar} \sum_{q}\left|\left\langle i^{\prime}\left|H_{e-p h}\right| i\right\rangle\right|^{2}\left(n_{q}+1\right) \delta\left(E_{i^{\prime}}-E_{i}+\hbar \omega_{q}\right)$

where $n_{q}$ is the phonon occupation number which is equal to 0 in the present calculation. The electron-LA phonon interaction Hamiltonian $H_{e-p h}$ is given by the following equation

$$
\begin{gathered}
H_{e-p h}=\sum_{i i^{\prime}} \sum_{q} V_{i i^{\prime}}(q) C_{i^{\prime}}^{\dagger} C_{i}\left(a_{q}+a_{-q}\right) \\
V_{i i^{\prime}}=D \sqrt{\frac{\hbar}{2 d \omega_{q}}}\left\langle i^{\prime}\left|q e^{i q r}\right| i\right\rangle
\end{gathered}
$$

where $C_{i}^{\dagger}\left(C_{i}\right)$ is an operator to create (annihilate) an electron of state, $i$ and $a_{q}^{\dagger}\left(a_{q}\right)$ is an operator for creation (annihilation) of a phonon with momentum $q$. In the present calculation we assume that the electrons interact with bulk LA phonons and thus the deformation potential for electron-LA phonon is given by the deformation potential of the bulk GaAs, $D=7 \mathrm{eV}$.

\section{RESULTS AND DISCUSSION}

Figure 2 shows the relaxation times for transition from the excited states to the ground state in a QD with $\hbar \omega_{0}=1 \mathrm{meV}$ and $W=50 \AA$. In the relaxation processes we have to take into account scattering
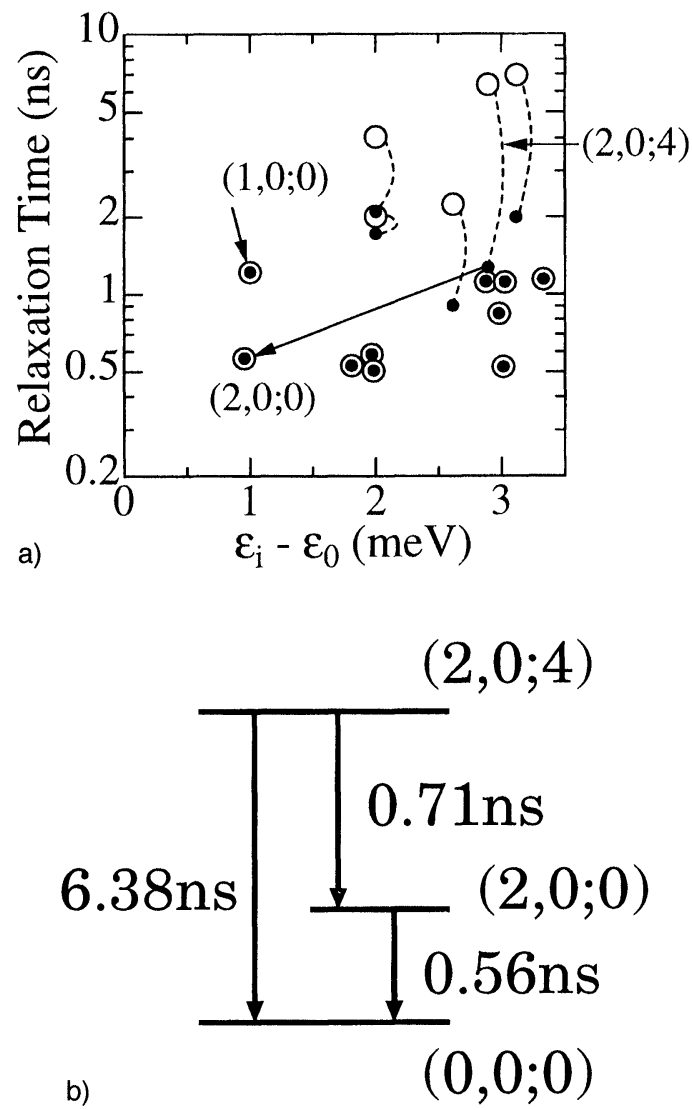

FIGURE 2 Relaxation time as a function of the energy difference between the initial state and the ground state for a two-electron QD (a). Large open circles and small solid circles denote the direct relaxation and the fastest relaxation, respectively. A schematic diagram for the direct and the fastest relaxations from the initial state $(2,0 ; 4)$ is also plotted (b).

from the excited state to the ground state through various intermediate states in addition to the direct transition. In Figure 2(a) we show the calculated relaxation time toward the ground state in a twoelectron QD where open circles denote direct processes and the fastest relaxations are plotted by the solid circles. The indices $(\mathcal{M}, \mathcal{S} ; \mathcal{N})$ shown in Figure 2 are quantum numbers of eigen states, indicating $\mathcal{N}$-th state of $N$-electrons system with a total angular momentum $\hbar \mathcal{M}$ and a total spin $\hbar \mathcal{S} / 2$. The connected pairs of an open circle and a solid circle indicate the relaxation from the same initial states and mean the existence of faster indirect process, whereas the open circles with the 
solid circles indicate that the fastest process is the direct process and the indirect process is very slow because of the symmetry of the wave functions. For electrons with $(\mathcal{M}, \mathcal{S} ; \mathcal{N})=(2,0 ; 4)$, the fastest indirect transition arises from the scattering process via the state $(2,0 ; 0)$ as shown by the arrow. A schematic diagram of the relaxations for the $(2,0 ; 4)$ state is illustrated in Figure 2(b), where the energy separations from the ground state and the relaxation times are given for the corresponding eigen states in Figure 2(a). The relaxation time for the direct process is about $6.38 \mathrm{~ns}$, whereas the relaxation time for the indirect process is given by $1.27 \mathrm{~ns}$ which is given by the sum of 0.71 ns for the scattering from $(2,0 ; 4)$ to $(2,0 ; 0)$ and $0.56 \mathrm{~ns}$ for the scattering from $(2,0 ; 0)$ to the ground state $(0,0 ; 0)$. In Figure 3 we plotted relaxation times for $N=3$ and 4 electrons in a QD with $\hbar \omega_{0}=1 \mathrm{meV}$ and $W=50 \AA$, where the electrons at higher energy states have longer relaxation time due to the difficulty in satisfying the energy and momentum conservation. Although energy levels become dense with increasing the number of electrons, modification of relaxation times are not so strong because many electron eigen states consist of only a few dominant single electron states which limit the electron relaxation for many electron states.

In Figure 4 we compare the fastest relaxation times in two QDs with different well widths $W=50$ $\AA$ (solid circles) and $100 \AA$ (open triangles), where two cases are considered, one for 2 electrons (a)

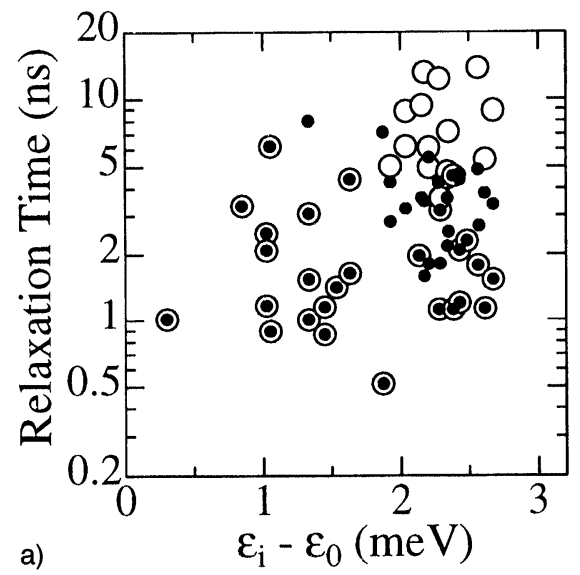

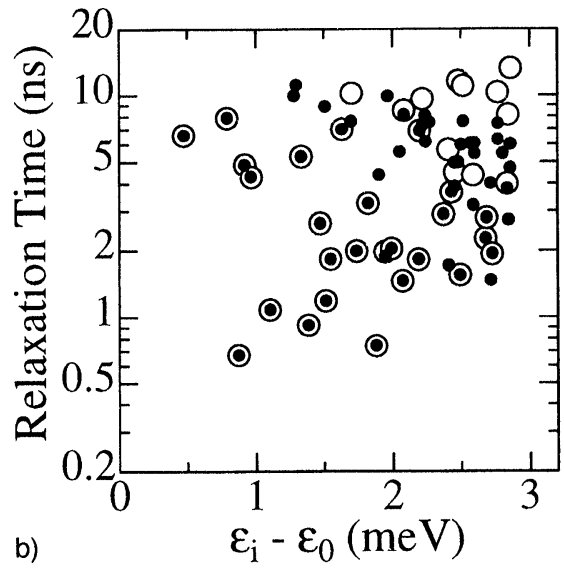

FIGURE 3 Relaxation time as a function of the energy difference between the initial state and the ground state for three (a) and four (b) electrons' QDs. Large open circles and small solid circles denote the direct relaxation and the fastest relaxation, respectively.

and the other for 3 electrons (b) in the QDs. The change in the well width $W$ results in the change in the confining energy in $z$ direction and in no change in the eigen energy in $x-y$ direction. The change in the confinement in the $z$ direction changes the maximum phonon wave vector $q_{\max }$ $\sim 2 \pi / W$ which gives the maximum phonon energy $\hbar c_{s} q_{\max }$ [8] where $c_{s}$ is the sound velocity. Maximum phonon energy is $4.3 \mathrm{meV}$ for $W=50 \AA$

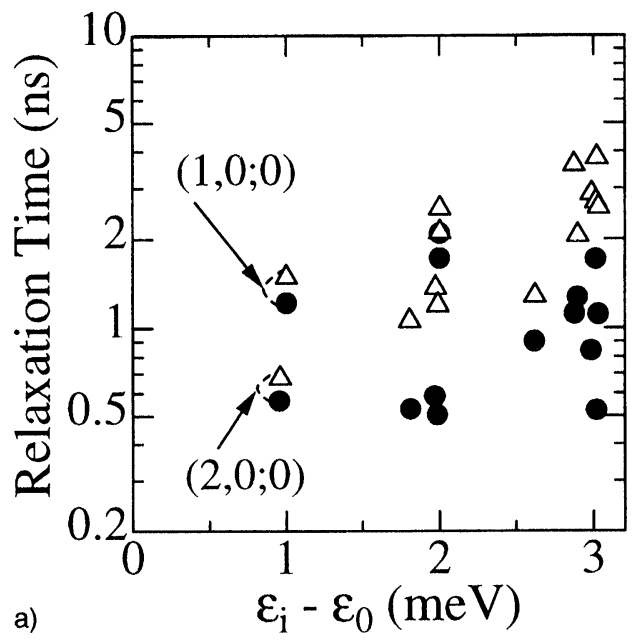

FIGURE 4 Electron relaxation rate in a QD containing the number of electrons $N=2$ (a) and 3(b) with different well widths of $W=50 \AA$ (solid circles) and $100 \mathrm{~A}$ (open triangles). 


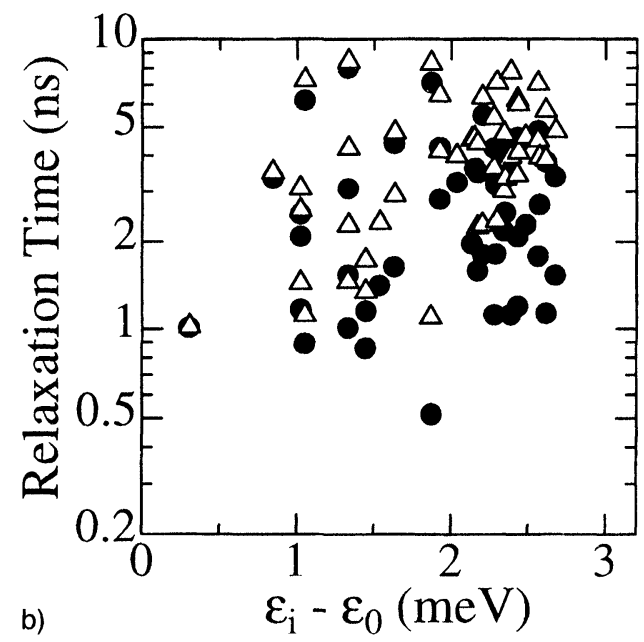

FIGURE 4 (Continued).

and is $2.2 \mathrm{meV}$ for $W=100 \AA$. It is seen in Figure 4 that the relaxation time for a QD with $W=100 \AA$ begins to increase at the energy separation of 2 $\mathrm{meV}$. From the present analysis it may be concluded that the change of the confining energy in the $z$ direction results in a change in the electron relaxation process even in a QD with several electrons.

\section{CONCLUSION}

Electron relaxations toward the ground state via LA-phonon scatterings in a QD with interacting $N$ electrons (from $N=1$ up to 4 ) are calculated using $N$ electrons' eigen states obtained by the exact diagonalization method. We find that energy levels become dense with increasing the number of electrons and that the modification of relaxation times is not so strong because many electron eigen states consist of only a few dominant single electron states which limit the electron relaxation. Several fastest processes are realized by relaxation through intermediate states between the initial state and the ground state.

We find that the confinement in the $z$ direction limits the maximum phonon energy and relaxation time becomes longer when an energy separation between the initial state and the final state exceeds maximum phonon energy.

\section{References}

[1] Ashoori, R. C., Stormer, H. L., Weiner, J. S., Pfeiffer, L. N., Baldwin, K. W. and West, K. W., " $N$-Electron Ground State Energies of a Quantum Dot in Magnetic Field", Phys. Rev. Lett., July 1993.

[2] Weinmann, D., Häusler, W. and Kramer, B., "Spin Blockades in Linear and Nonlinear Transport through Quantum Dots", Phys. Rev. Lett., February 1995.

[3] Tarucha, S., Austing, D.G., Honda, T., van der Hage, R. J. and Kouwenhoven, L. P., "Shell Filling and Spin Effects in a Few Electron Quantum Dot", Phys. Rev. Lett., October 1996.

[4] Bazán, G., Orlov, A. O., Snider, G. L. and Bernstein, G. H., "Charge detector realization for $\mathrm{AlGaAs} / \mathrm{GaAs}$ quantum-dot cellular automata", J. Vac. Sci. Technol. B, Nov./Dec. 1996.

[5] Buks, E., Schuster, R., Heiblum, M., Mahalu, D., Umansky, V. and Shtrikman, H., "Measurement of Phase and Magnitude of the Reflection Coefficient of a Quantum Dot", Phys. Rev. Lett., November 1996.

[6] Benisty, H., Sotomayor-Torrès, C. M. and Weisbuch, C., "Intrinsic mechanism for the poor luminescence properties of quantum-box systems", Phys. Rev. B, November 1991.

[7] Bockelmann, U. and Bastard, G., "Phonon scattering and energy relaxation in two-, one-, and zero-dimensional electron gases", Phys. Rev. B, November 1990.

[8] Benisty, H., "Reduced electron-phonon relaxation rates in quantum-box systems: Theoretical analysis," Phys. Rev. $B$, May 1995 .

[9] Sotomayor Torres, C. M. (1996). "Energy Relaxation in quantum dots: recent developments on the phonon bottleneck", Hot carriers in Semiconductors, New York, Plenum Press.

[10] Sawaki, N., Niwa, S., Taya, M., Murakami, T. and Suzuki, T. (1996). "Reduction of energy relaxation rate of photo-excited hot electrons in quasi-one and zero dimensional structures", Hot carriers in Semiconductors, New York, Plenum Press.

[11] Bockelmann, U. and Egeler, T., "Electron relaxation in quantum dots by means of Auger processes", Phys. Rev. $B$, December 1992 .

[12] Inoshita, T. and Sakaki, H., "Electron relaxation in a quantum dot: Significance of multiphonon processes", Phys. Rev. B, September 1992.

[13] Bockelmann, U., "Exciton relaxation and radiative recombination in semiconductor quantum dots", Phys. Rev. B, December 1993.

[14] Maksym, P. A. and Chakraborty, T., "Quantum Dots in a Magnetic Field: Role of Electron-Electron Interactions", Phys. Rev. Lett., July 1990.

[15] Maksym, P. A. and Chakraborty, T., "Effect of electronelectron interactions on the magnetization of quantum dots", Phys. Rev. B, January 1992.

[16] Yang, R. and Ruden, P. P., "Electron-electron interaction in three-dimensional model quantum box", J. Appl. Phys., August 1995. 


\section{Authors' Biographies}

Tatsuya Ezaki is a graduated student at Department of Electronic Engineering, Osaka University, Osaka, Japan and expected to receive $\mathrm{Ph}$. D. degree in March 1998. He has been involved with research work on electron transport in quantum wires and infra-red detectors utilizing intersubband transition in multi-quantum wells. His current interests include electronic states in quantum dots and electron-phonon interaction in quantum dots. He is a memeber of Japan Society of Applied Physics.

Nobuya Mori is an Assistant Professor at Department of Electronic Engineering, Osaka Unviersity, Osaka, Japan. His research interests include quantum transport in semiconductor nano-structures and magnetotransport in semiconductors. He was a British Council Fellow at Department of Physics, University of Nottingham,
Nottingham, England, from August 1994 to March 1996. He is a member of Institute of Physics, a memebr of Physical Society of Japan, and a memebr of Japan Society of Applied Physics.

Chihiro Hamaguchi is a Professor of Electronic Engineering at Osaka Univesity, Osaka, Japan. His research interests include electron transport in semiconductor quantum structures, magnetotransport in semiconductors, modualtion spectroscopy and device simulation. He is a Fellow of the IEEE, a Fellow of the Americal Physical Society, Chairman of Electron Devices Society of IEEE Tokyo Chapter, Editorial Board of Semiconductor Science and Technology. $\mathrm{He}$ has served the chairman of International Conference on Hot Carriers, vice-chariman of International Symposium on New Phenomena in Mesoscopic Structures and committee member of many international conferences. 

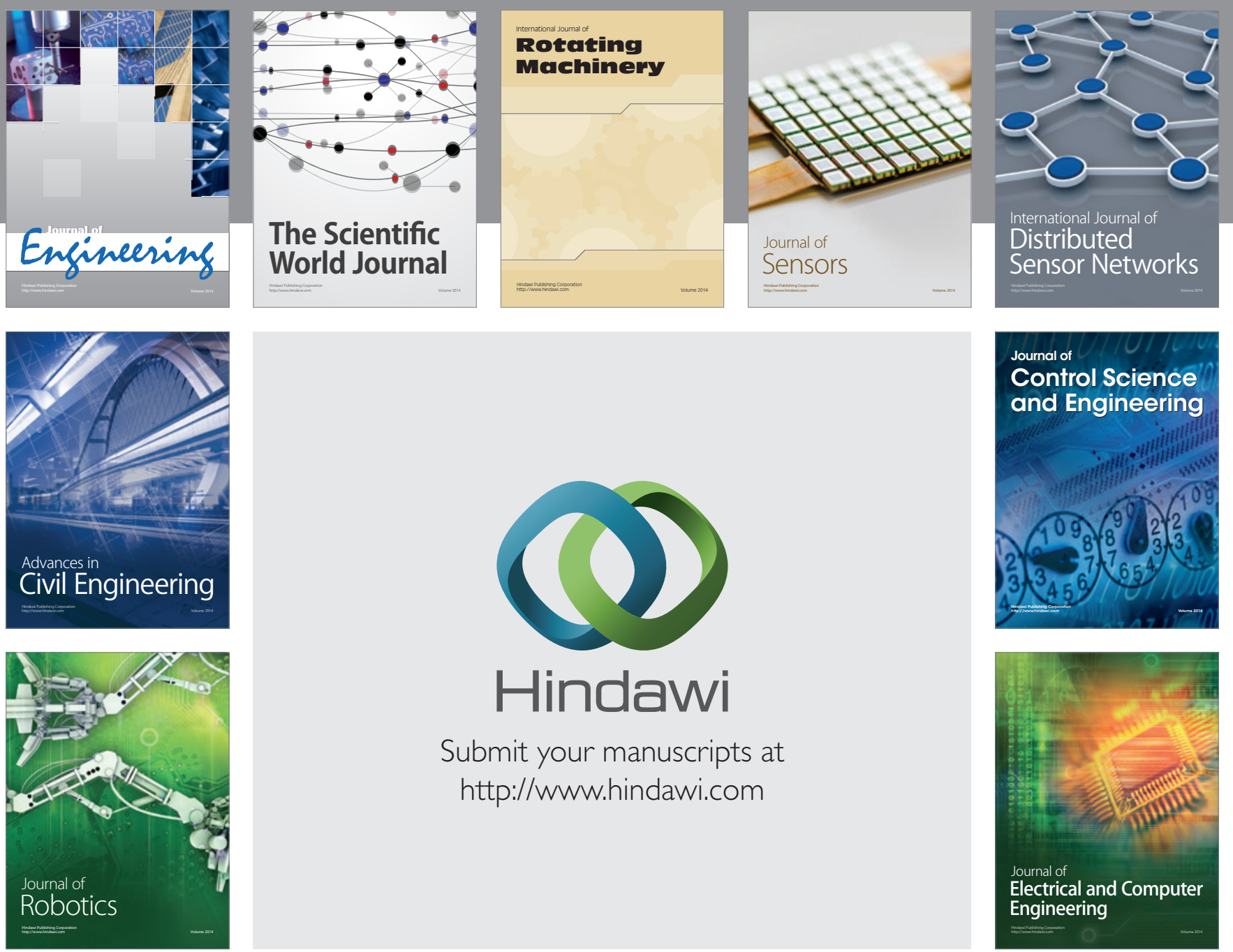

Submit your manuscripts at

http://www.hindawi.com
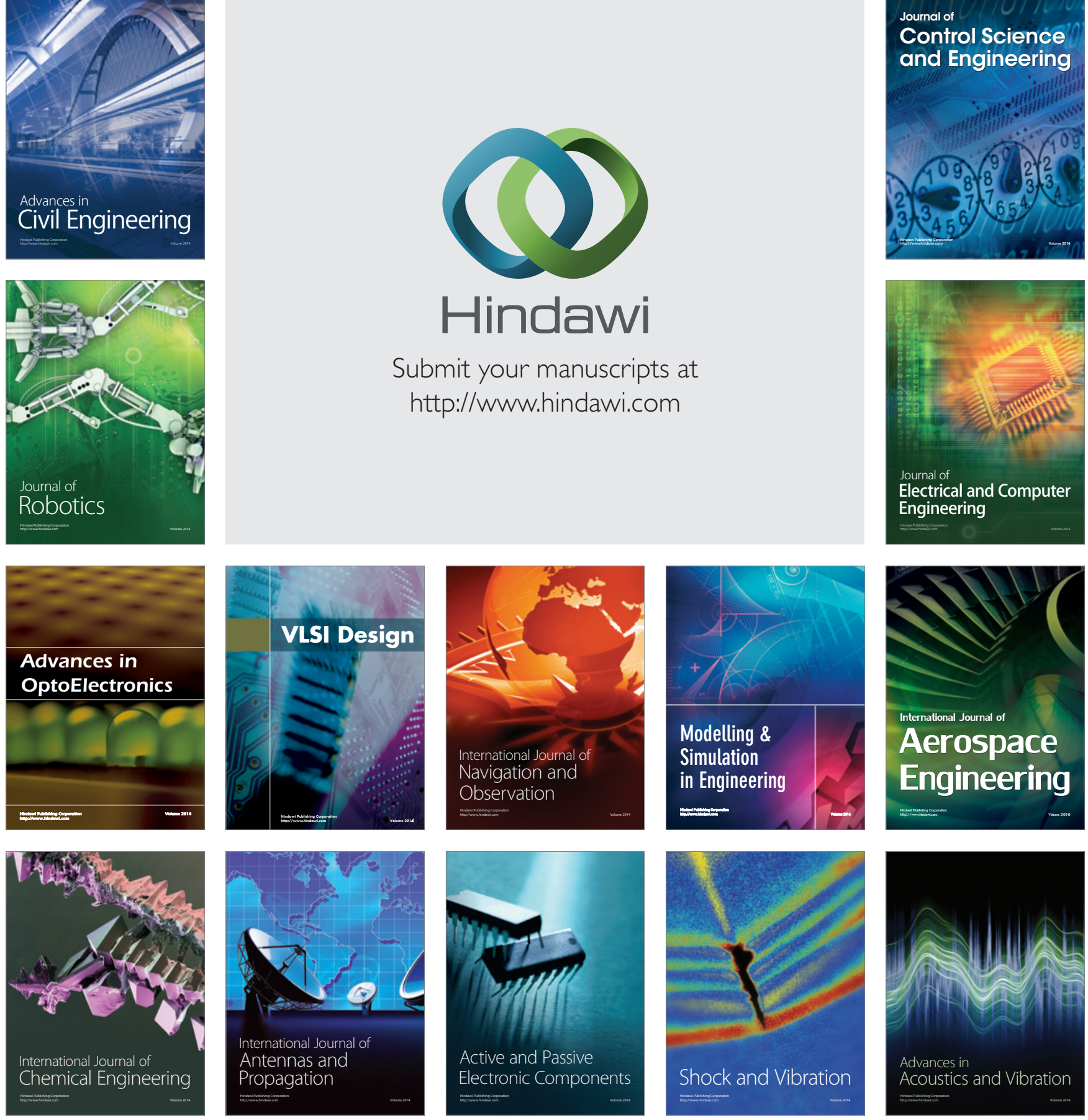